\title{
Review of: "A Comparison of Macaque Hair Hormone Concentration Following Enhanced Cognitive Experiences or Standard Nonhuman Primate Environmental Enrichment"
}

\author{
Darya Abdulateef ${ }^{1}$
}

1 University of Sulaimani

Potential competing interests: The author(s) declared that no potential competing interests exist.

Generally this study is a novel one, l'd like to thank you for the authors for choosing this subject, the physiological assessment of subjects within these different environment is needed which is not studied well previously or inconsistent results exists.

Although there are some shortcomings; for example the sample size is small especially when they divided one of the groups further into MR-ECE and NR-ECE, some problems in the writing and flow of the Introduction and minor note on the Figures that I had noticed were addressed below;

\begin{abstract}
Well written and comprehensive.
Introduction: The introduction is too long, and too much detail and repetition of the paragraphs with the same meaning were included which better to be in the discussion section rather than introduction. Usually, in the introduction three main paragraphs or subjects is needed; what is already known about the subjects, what is not known and should be investigated and the aims of the study. While in this Manuscript's Introduction the author at many paragraphs was going to finalize the introduction section by discussing what is not found and what is the aim of the study and what they did, but finally starting to discuss another subject again and continue to adding more new information.
\end{abstract}

Recommendations: It is better to edit the Introduction section by removing the unnecessary or repeated paragraphs, and correct the flow of the paragraphs to make the readers not confused. It is true in the introduction section you have to discuss what is known about the subjects, but no need to address all literatures, only if it is introduction and literature review. Also, no need to discuss what you did in your research for example how you grouped your animal, this is better to be discussed in the method section rather than introduction.

We also recommending to address all what known about ECE \& CAU, MR and NR, and Cortisol and other analyte use for stress and assessment of ... then try to discuss your aims all together and stating that no studies assess these values rather than repeating these sentences in three areas inside your introduction. 
Methods: The method section is written in an organized and detailed way, which is important for the method section to discuss all processes and every point in detail for someone to repeat the study. Try to add exclusion criteria to the method section. It is written one with outlier or more than 3SD from mean were excluded, but other points important to be discussed, whether monkeys with certain diseases, abnormalities or previous psychological problems, trauma or life stressor were excluded in the study or not.

I wonder whether the mentioned animal with inhibition of gonadal and adrenal hormones 3 years prior to hair sample collection was excluded from the study or not, if not why it is not excluded, please? For the assay method, I couldn't find the detail about LC/MS process or assay method in detail in the reference that you cited and claimed to do it according to the what described there, could you please add a few sentences on the way LC/MS were performed and the results read and recorded, whether any calculation or unit conversion is needed, is their use of any other materials?

I would like to ask if the use of unground hair is more practical with LC/MS method, as in the analysis of hair cortisol in most studies with ECLIA the hair were cut or grounded to give better exposure and extraction of cortisol from the hair.

Finally, I recommend if available add an image for both ECE and CAU environment that they used in their study.

I recommend using Care as usual environment rather than Case as usual enrichment, because the term enrichment make the reader to confuse and think of the an enriched environment.

\section{Results:}

The results are demonstrated and defined well, while I'm not sure if the finding of less inter-individual variability in these analytes are important to assess and addressed and if they indicate a better stress response. Could you provide few references, please?

The sequences of the Figure legends are incorrect, not comply with the sequences of the Figures, although I didn't see the Figure captions on the images. For example Figure 1 legend is describing the third sequence Figure.

For the both Figures of scatterplots, it is better to draw the lines in order to be differentiated, which line is of ECE-MR and which one is of ECE-NR, one of them dashed line and one of them solid line and define them like how you defined the circles. And P-value should be added on the Figure whether this correlation is significant or not you have to add the exact P-values. For the statistic that used to find these correlations, I think Spearman's correlations were used as your data were not distributed normally, but you stated that regression analysis were done, while no detailed results from regression were added, what I can see just simple correlation which are shown with scatterplots.

The units of the cortisol and DHEA better to be added on the Figures.

\section{Discussion and conclusion:}


They are generally good, but try to not overestimate your finding. In the third paragraph of the Discussion section, page 16, second line, "Our observations are consistent with the findings of previous studies which demonstrated that acute experience with ECE lowers salivary cortisol" I think you can discuss this study finding to support yours, but you couldn't say consistent to our study as their results give lower cortisol level in ECE, while in the current study the comparison of analytes between groups are not significant. This sentence in the discussion section (page 17, first paragraph) is not needed,

"Additionally, the effects of glucocorticoids are influenced by many factors, such as the amount of free glucocorticoids and amount of glucocorticoid receptors" because one of the benefit of assessing cortisol in hair over other method is that, hair cortisol will give the free hormone.

This statement in the same page, is also not clear to me "we are confident in our interpretation that provision of EDE improve NHP welfare, .." It is also not clear whether this is your statement or other study's statement as references were cited.

References: The use of numbering style in the references (Vancouver and others) were more common than citing author names and years in the text (Harvard), and will lead to less content and distractions during reading, although the Journal style is important to follow. 